\title{
Ultra-low contrast coronary angiography and zero-contrast percutaneous coronary intervention for prevention of contrast-induced nephropathy: step-by-step approach and review
}

\author{
Jerzy Sacha ${ }^{1,2}$, Marek Gierlotka ${ }^{1}$, Piotr Feusette ${ }^{1}$, Dariusz Dudek ${ }^{3}$ \\ ${ }^{1}$ Department of Cardiology, University Hospital, Faculty of Natural Sciences and Technology, University of Opole, Opole, Poland \\ ${ }^{2}$ Faculty of Physical Education and Physiotherapy, Opole University of Technology, Opole, Poland \\ ${ }^{3}$ Institute of Cardiology, Jagiellonian University Medical College, Krakow, Poland
}

Adv Interv Cardiol 2019; 15, 2 (56): 127-136

DOI: https://doi.org/10.5114/aic.2019.86007

\begin{abstract}
A bstract
Contrast-induced nephropathy is a serious complication after intravascular administration of iodinated contrast media and is associated with numerous adverse outcomes. Its prevalence is particularly high in patients with multiple comorbidities who undergo coronary angiography and percutaneous coronary intervention $(\mathrm{PCI})$. Currently, the only effective method to prevent contrast-induced kidney injury is adequate hydration and a reduction of contrast volume during the intervention. Recently, new approaches aiming to minimize contrast usage have been proposed, i.e., ultra-low contrast angiography and zero-contrast PCI. However, neither tutorials for these techniques nor reviews of their outcomes exist in the literature, and therefore dissemination of these approaches among the interventional community may be limited. This article presents a step-by-step description on how to perform ultra-low coronary angiography and zero-contrast $\mathrm{PCl}$, which should help invasive cardiologists to adopt these techniques in daily practice. A review of clinical studies, case series and single case reports regarding these methods is also provided. Despite the promising results, such procedures still require some improvements and confirmation of their effectiveness as well as safety in large clinical studies. This article aims to spread these new techniques throughout the interventional community, which is paramount for their further development and wider utilization.
\end{abstract}

Key words: renal insufficiency, chronic kidney disease, contrast-induced nephropathy, acute kidney injury, zero-contrast percutaneous coronary intervention.

S u m m a ry

Contrast-induced nephropathy (CIN) is a common complication after diagnostic and therapeutic coronary procedures and is independently associated with adverse outcomes. Recently, approaches aiming to minimize contrast dose during the interventions have been proposed which appear to be a promising preventative strategy against CIN. This article provides a step-by-step description on how to perform ultra-low coronary angiography and zero-contrast percutaneous coronary intervention, and reviews up-to-date clinical data concerning these new techniques.

\section{Introduction}

Contrast-induced nephropathy (CIN) is an acute renal impairment that may appear within 48-72 h after intravascular administration of iodinated contrast media and is defined as an increase in the serum creatinine level of more than $0.5 \mathrm{mg} / \mathrm{dl}(44 \mu \mathrm{mol} / \mathrm{l})$ or an increase of at least $25 \%$ in the level from baseline [1]. CIN particularly affects subjects with underlining chronic kidney disease (CKD), diabetes, heart failure, acute coronary syndrome and cardiogenic shock, and it is associated with progression of renal failure, necessity of dialysis, prolonged hospitalization, increased costs and mortality [1-3]. It has been shown that intra-arterial compared with intravenous contrast media administration may be linked to a greater risk of $\mathrm{CIN}$, although the mechanism of this

Corresponding author:

Jerzy Sacha MD, PhD, Department of Cardiology - University Hospital in Opole, Al. Witosa 26, 45-401 Opole, Poland, e-mail: sacha@op.pl Received: 2.12.2018, accepted: 1.04.2019. 
phenomenon is not clear [4, 5]. Patients undergoing coronary angiography and percutaneous coronary intervention ( $\mathrm{PCl}$ ) present an increased risk of CIN especially in the setting of urgent intervention [2, 3]. During such procedures, large amounts of contrast media are usually administered and a strong relationship exists between contrast volume and a risk of $\mathrm{CIN}[2,3]$. Adequate hydration with intravenous saline infusion before and after intervention is a key preventative measure against CIN [1]. Recently, new strategies dedicated to the reduction of contrast volume have been proposed, and these appear to be promising approaches in the battle against CIN [6-12]. Such low-amount contrast procedures may improve patients' outcomes, and therefore deserve dissemination within the interventional and wider medical communities. In this article, we present step-by-step principles on how to perform ultra-low contrast coronary angiography and zero-contrast $\mathrm{PCl}$, and we review up-todate evidence on feasibility, safety and clinical outcomes of such approaches.

\section{Catheter engagement without contrast administration}

Both femoral and radial accesses are acceptable for ultra low- or zero-contrast techniques, but patients with advanced renal failure present a high risk for renal replacement therapy, and therefore radial arteries should be saved for arteriovenous fistula in case of chronic hemodialysis. In most instances, the catheter engagement without contrast injection does not pose a problem for an experienced operator. Patients with renal diseases usually present calcified lesions in proximal parts of their coronary arteries [13], and a detailed analysis of the calcium distribution may help to identify the arteries and guide the catheter engagement. High frame rate acquisition (i.e., 30 frames/s) exposes calcified changes and greatly facilitates identification of the vessels. To confirm the proper engagement, one should inject 10-20 ml of saline through the catheter and observe temporal changes in the electrocardiogram [14]. If the catheter is properly inserted, the saline injection induces the T-wave inversion or increases its amplitude along with ST-segment depression or elevation (Figure 1). One may also confirm the appropriate catheter position by introducing a coronary guidewire into the artery, i.e., if the guidewire follows the anatomy of the coronary artery then this is the confirmation of proper engagement. However, this method requires heparin administration and poses some risk of dissection when coronary lesions are not known; therefore, it is usually employed in the setting of $\mathrm{PCl}$.

\section{Ultra-low contrast coronary angiography}

In the ultra-low contrast technique, a reliable coronary angiography may be performed by using less than

A Left coronary artery $\sim \sqrt{ } \sqrt{2} \sqrt{2}$ aVR $10 \mathrm{~mm} / \mathrm{mV}$

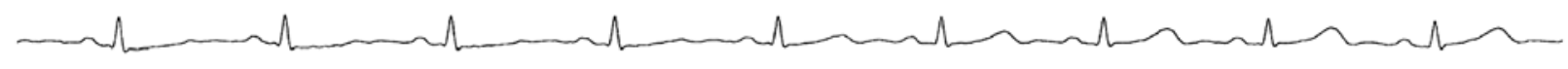

$10 \mathrm{~mm} / \mathrm{mV}$

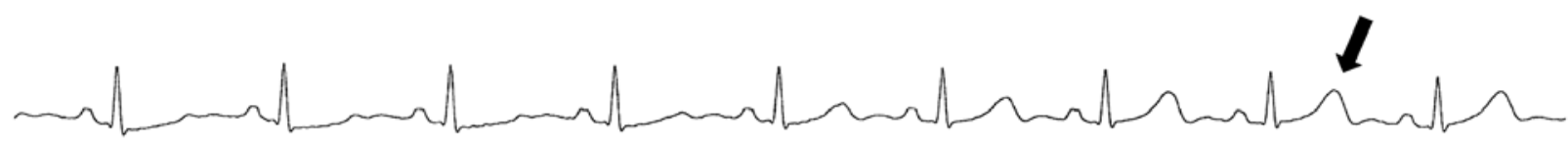
$1 \mathrm{~mm} / \mathrm{mV}$

B Right coronary artery
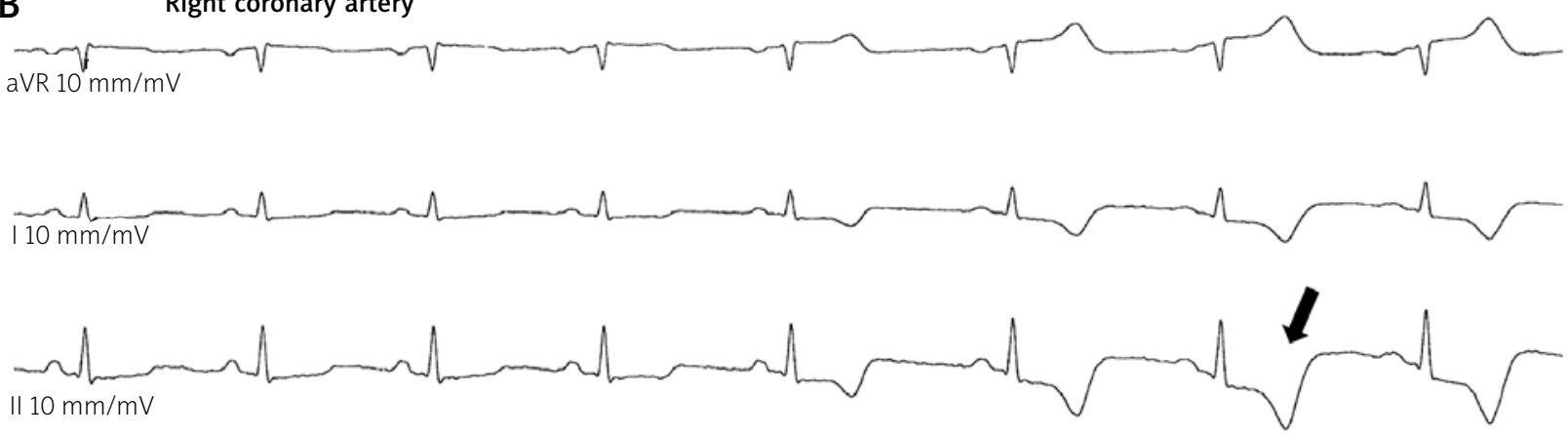

Figure 1. Electrocardiographic changes during an injection of $20 \mathrm{ml}$ of saline into the catheter confirm proper engagement. A - Left coronary artery (arrow indicates T-wave increase). B - Right coronary artery (arrow indicates ST-segment depression with T-wave inversion) 
$15 \mathrm{ml}$ of contrast medium [6]. A nonionic, iso-osmolar contrast agent is preferred, and its pre-specified maximum volume should be based on the ratio of contrast volume (CV) per estimated glomerular filtration rate (eGFR) - when the ratio (i.e., CV/eGFR) is less than 1 , the risk of CIN is significantly reduced (e.g., if the patient's eGFR equals $20 \mathrm{ml} / \mathrm{min} / 1.73 \mathrm{~m}^{2}$, the contrast volume should not exceed $20 \mathrm{ml}$ ) [15-17]. Administration of 2-3 ml of contrast is usually sufficient to visualize the left coronary artery, but $2 \mathrm{ml}$ (or even less) is enough for the right coronary artery. Such low-dose contrast injections should be performed through small diameter catheters (i.e., 5-6 Fr) without side-holes and with small syringes (e.g., 3 or $5 \mathrm{ml}$ ) to enable fast and easy administration (dye "puffing" should be avoided).

Usually, the arterial tree appears sequentially as the low-contrast volume flows from its proximal to distal portion; therefore, the acquisition time should be appropriately adjusted, with a high frame rate being preferred (i.e., 30 frames/s) since it significantly improves vessel visualization. Before any drug administration (e.g., nitroglycerine) or when exchanging catheters, contrast medium must be removed from the catheter to avoid pushing it into the patient. Depending on the type of catheter, manifold and $Y$ connector, the volume contained in the whole system may range from 1.6 to more than $4 \mathrm{ml}-$ Table I presents examples of fluid volumes filled in different catheters and systems. After drug delivery, the catheter must be refilled with 1-3 $\mathrm{ml}$ of contrast (depending of the catheter size) before cine angiography, otherwise the administration of a small dye volume may result in insufficient opacification of the artery, necessitating a repeated injection.

The left coronary artery angiography usually begins from the spider view and then the cranial right anterior oblique projection is acquired; other projections depend on lesions' distribution and visualization. For right coronary angiography, the cranial left anterior oblique projection is usually sufficient. Optionally, acquisition with the right anterior oblique projection may be performed. If more projections are necessary, contrast dilution with saline $2: 1$ may limit the overall contrast amount. However, a higher dilution (e.g., 1 : 1) may yield unsatisfacto- ry images and usually requires repeated administration. Another way to reduce the contrast dose is a biplane angiography, which limits the number of acquisitions and has been proven to mitigate the risk of CIN without increasing radiation exposure [18]. However, many catheterization laboratories are not equipped with such biplane devices, and therefore this tutorial is dedicated to a single plane angiography, which may be easily adopted for a biplane version.

\section{Zero-contrast percutaneous coronary intervention}

If angiographic images of coronary lesions are known, one may attempt to perform $\mathrm{PCI}$ without contrast administration. Such interventions are mainly guided by intravascular ultrasound imaging (IVUS), which helps to identify the lesion and its length (i.e., "normal-to-normal" distance), reference vessel diameters and landing zones for stent implantation [6, 7]. However, the first step is to carefully analyze the anatomy of arteries in previous coronary angiography with particular consideration of calcified plaques. Such calcified changes may reflect the coronary tree without angiography and often indicate the position of the target lesion - a higher acquisition frame rate helps to expose these calcifications. The same projections that were employed during the angiography must be used for $\mathrm{PCl}$, and previous angiographic images should be displayed alongside the active fluoroscopy screen as a reference. Larger guide catheters are helpful (usually $7 \mathrm{Fr}$ ) since they provide better support and may accommodate several guidewires along with the IVUS probe or stents. During a meticulous insertion, the guidewire should exactly follow the course of the vessel displayed on the reference screen, and attention should be paid to every side branch in order to avoid accidental wiring. In general, workhorse guidewires without hydrophilic coated tips are preferred to mitigate the risk of vessel perforation. It is useful to create an excessive curve at the tip of the wire so that the tip will form a loop after crossing the stenotic lesion, and by pushing such a "knuckled" wire (without rotating!) one may safely insert it even throughout a tortuous vessel. Such a loop usually follows the main vessel and omits side branch-

Table I. Contrast volumes contained in different diagnostic and guide catheters connected to manifold (first port) or standard $\mathrm{Y}$ connector (22 cm tube and 3-way stopcock)

\begin{tabular}{lccc}
\hline Catheter & Catheter alone $[\mathrm{ml}]$ & Catheter with manifold $[\mathrm{ml}]$ & Catheter with $\mathrm{Y}$ connector $[\mathrm{ml}]$ \\
\hline Diagnostic $5 \mathrm{Fr}$ & 1.3 & 1.6 & 2.6 \\
\hline Diagnostic 6 Fr & 1.6 & 1.9 & 2.9 \\
\hline Guide 6 Fr & 2.6 & 2.9 & 3.9 \\
\hline Guide 7 Fr & 3.3 & 3.6 & 4.6
\end{tabular}

Since blood flow elutes some contrast from the end of the catheter, the real contrast volume contained in the above systems is approximately 0.5-1.0 ml less. The inner diameters of the catheters were as follows: diagnostic - 0.05 " ( 5 Fr); 0.57 " (6 Fr); and guide - 0.71 " (6 Fr); 0.081 " ( $7 \mathrm{Fr}$ ). 
es; moreover, it also prevents vessel perforation during $\mathrm{PCl}$. For confirmation of proper guidewire insertion, one should compare the reference image taken from the previous angiography with the actual course of the wire. If the two images correspond, then the IVUS examination can be performed. When the position of guidewire is correct, but the IVUS probe cannot cross the stenotic lesion, its location should be recorded with cine angiography and such an image will provide a reference for balloon pre-dilation. After pre-dilation, a repeated IVUS examination should determine the lesion length, vessel diameter and landing zones for stent implantation. The first non-diseased segment behind the lesion (or a segment with plaque burden less than $50 \%$ in cases with diffuse lesions) constitutes the distal landing zone, and importantly, the corresponding IVUS probe position should be recorded. The same has to be done for the proximal landing zone. It is crucial to seek landmarks that may indicate the location of the landing zones. The most helpful landmarks are calcifications within vessels and the chest wall, overlying the ribs, diaphragm, surgical clips (in patients after coronary artery bypass grafting) as well as the catheter position, which all together may assist in proper stent positioning and implantation [7, 10, 19]. Figure 2 shows an example of how to take advantage of anatomical landmarks during zero-contrast PCl. It should be stressed that due to the heart motion and respiration, the landing zones are usually moving in reference to the landmarks. Hence, one needs to carefully analyze the features of these movements and accordingly cover such "moving landing zones" with the stent. Holding respiration during stent implantation should be avoided since a deep inspiration may significantly change the position of the entire system. After stent placement, IVUS imaging ascertains stent expansion and may reveal edge dissection. However, IVUS is not able to recognize vessel perforation and distal embolization. Therefore, if there is any doubt about the proper guidewire insertion or presence of complications, one should fill the guide catheter with $3 \mathrm{ml}$ of contrast and then inject only $0.5-1.0 \mathrm{ml}$ to elucidate the problem. Afterwards, the unused contrast must be withdrawn from the catheter (Table I).

It should be mentioned that almost all interventional techniques can be employed during zero-contrast PCI. Particularly, physiological assessment of coronary lesions with pre- and post- $\mathrm{PCl}$ measurements of fractional flow reserve (FFR) and coronary flow reserve (CFR) turned out to be very helpful for both procedure guidance and confirmation of physiological improvement after intervention [7]. However, by using these techniques one needs to wire the vessel pre- and post- $\mathrm{PCl}$, which may pose a risk of dissection. Yet, by holding the workhorse wire in the vessel and with the help of a double lumen microcatheter, one may insert a pressure wire several times and do pull-back measurements with minimal risk of vessel damage. Also by using a single lumen microcatheter one may safely exchange the operating wire for a pressure wire and vice versa. Another option is a rapid-exchange microcatheter-based pressure measurement system (ACIST RXi System, ACIST Medical Systems), which eliminates the need for a wire exchange and provides a safer and more rapid assessment of FFR.

Rotational atherectomy is also feasible without contrast administration, since calcified plaques usually indicate both the location of the culprit lesion and the extent of the vessel that needs to be ablated. However, this procedure can only be done in selected cases with non-complex coronary anatomy [9]. One of the problems that may occur during rotational atherectomy is a drop in blood pressure, which suggests complications, e.g., vessel rupture or distal embolization. If the pressure drop persists, an injection of a small amount of contrast may exclude life-threatening complications. Other devices, such as embolic protection devices or thrombus aspiration catheters, can also be used if the coronary artery anatomy is known and the vessel has been thoroughly explored with IVUS imaging [19, 20].

To adopt the technique of zero-contrast $\mathrm{PCl}$ in a catheterization laboratory, it is reasonable to establish a local training program. In such a program operators should train the guidewire insertion and balloon as well as stent positioning without contrast usage during standard PCls (i.e., under ultimate control of contrast injections), and for maintenance of this skill, they should repeat such procedures periodically. In addition, they ought to implement ultra-low contrast coronary angiographies in daily practice.

\section{Techniques for procedure navigation}

During zero-contrast interventions, the most challenging task is proper stent positioning according to the landing zones determined in IVUS. The anatomical landmarks described above (particularly calcifications within vessels) are crucial in the navigation of such a procedure. However, several other techniques have been proposed to provide additional assistance during the operation. One of these techniques is the so-called "marking wire technique", where two wires are inserted into the coronary artery through a double $Y$ connector: the operating wire is put in via the main port but the marking wire via the side port of the connector (Figure 3) [11]. The marking wire serves as a guide for stent positioning, i.e., its tip is placed at the level of the distal landing zone determined by IVUS and then its position is secured by screwing the connector's side port and by attaching a torquer (Figures $3 \mathrm{~A}-\mathrm{C}$ ). With such a marking wire, one may subsequently determine (by using IVUS) the proximal landing zone and the stent length (Figure $3 \mathrm{D}$ ). Finally, the stent is positioned and implanted according to the location of the marking wire (Figures $3 \mathrm{E}, \mathrm{F}$ ). The whole system 

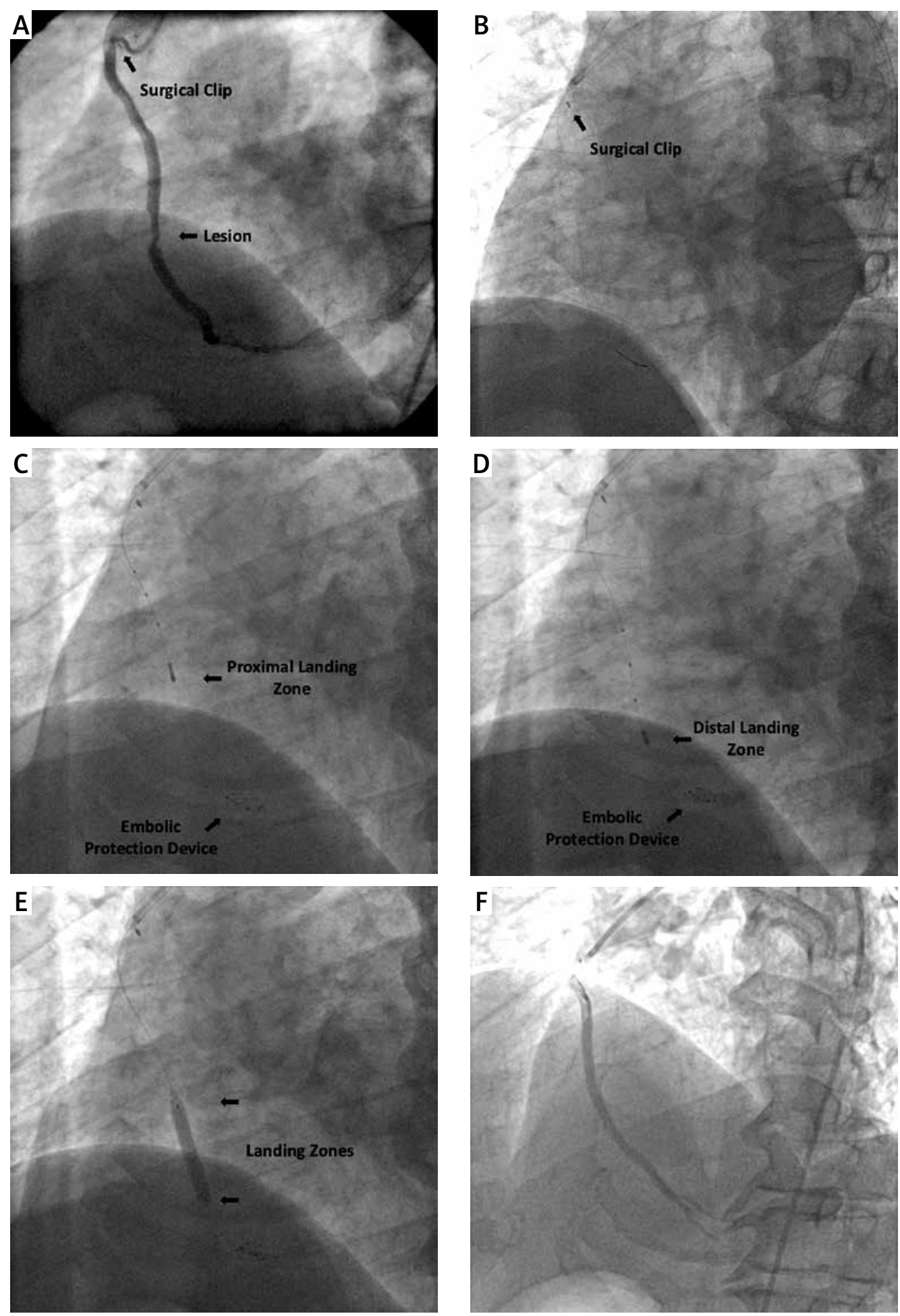

Figure 2. Zero-contrast percutaneous coronary intervention (PCI) guided by anatomical landmarks. A - Angiography in a patient with renal dysfunction, performed 4 days before $\mathrm{PCl}$, reveals $80 \%$ stenosis in middle portion of saphenous vein graft (SVG) to right coronary artery (RCA) (upper arrow indicates a surgical clip of other graft which superimposes on the ostium of SVG-RCA). B - During staged PCl, a guide catheter was engaged at the ostium of SVG-RCA according to the position of the overlying surgical clip - correct catheter placement was confirmed by inserting the guidewire into the vessel. C - Intravascular ultrasound (IVUS) identified the lesion and determined the proximal landing zone for stent implantation in reference to the ribs. $\mathbf{D}$ - The distal landing zone was determined at the lower edge of the rib. $\mathbf{E}$ - The stent was implanted in reference to the ribs. $\mathbf{F}$ - Due to legal issues, a single contrast injection documented the final PCI result. Reprinted from Sacha and Feusette [19] 

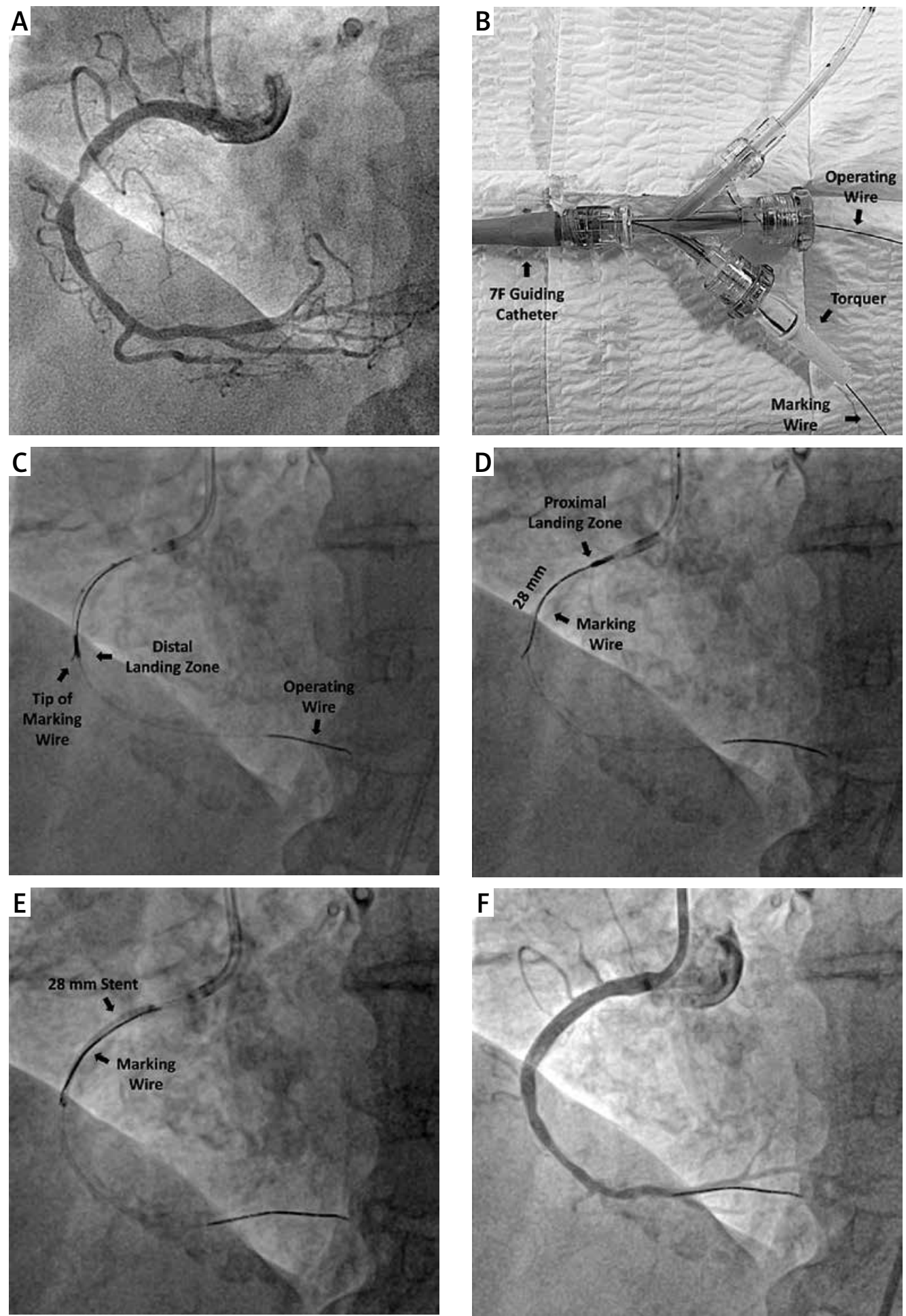

Figure 3. Marking wire technique for zero-contrast percutaneous coronary intervention (PCI). A - Right coronary artery (RCA) angiography in a patient with renal impairment performed several days prior to $\mathrm{PCI}$. B - During staged PCl, a double $\mathrm{Y}$ connector was attached to the guide catheter and two guidewires were inserted, i.e., the operating and marking wires. C - The distal landing zone was marked with the tip of the marking wire according to the position of the intravascular ultrasound (IVUS) probe. D - The proximal landing zone was determined by IVUS, and the stent length was estimated with the help of a marking wire. $\mathrm{E}$ - The stent was positioned according to the marking wire. $\mathbf{F}$ - Due to legal issues, a single contrast injection documented the final PCI result. Reprinted from Sacha [11] 

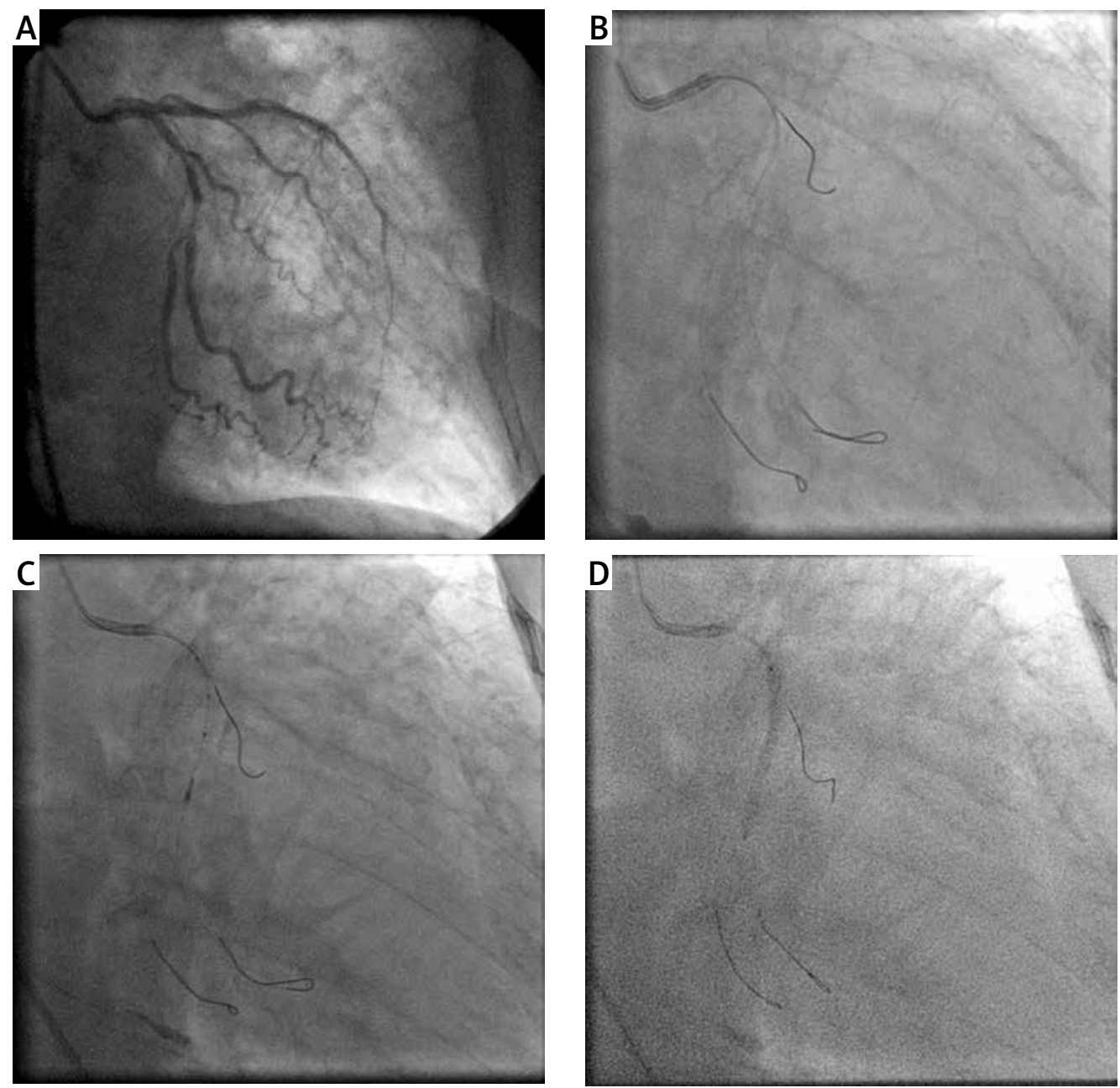

Figure 4. Zero-contrast percutaneous coronary intervention $(\mathrm{PCl})$ with construction of a metallic silhouette of the vessel. A - Left coronary angiography performed several days prior to $\mathrm{PCl}$ shows significant stenoses within circumflex artery (CX). B - The metallic silhouette of Cx is created by inserting guidewires (through a $7 \mathrm{Fr}$ guide catheter) into the main branch and two side branches - for safety reasons, the tips of wires should be loop shaped. C - Intravascular ultrasound (IVUS) identifies reference diameters and landing zones for stent implantation. D - Coronary stent implantation according to the position of the side branch guidewires

is very stable and the balloon pre-dilation or insertion of the IVUS probe usually does not change the marking wire position - although for its stability, the diameter of the guide catheter should not be smaller than $7 \mathrm{Fr}$. The marking wire technique facilitates zero-contrast $\mathrm{PCl}$ particularly in cases where the artery is tortuous or very mobile, and therefore the landing zones for stent implantation are very hard to identify. This technique may also reduce the stent length, which is usually longer in $\mathrm{PCl}$ without contrast.

Another technique that helps in orientation within a coronary artery relies on building a metallic silhouette of the vessel with guidewires [7, 8]. Side branches often provide important landmarks for stent implantation, and by wiring these branches along with the main vessel, one may create a metallic silhouette of the artery. Akin to the case of the main vessel, it is reasonable to wire side branches with loop-shaped tip guidewires. To do this, an excessive curve at the tip of the wire should be created, and after entering the side branch, one should allow the guidewire to form a loop. By pushing such a guidewire without rotating, one can insert it to a further portion of the vessel. In this way, a silhouette of the coronary system is created, which constitutes a useful road map for the intervention (Figure 4). Moreover, such wires also secure side branches from occlusion during $\mathrm{PCl}$.

Recently, new techniques of angiographic co-registration of imaging modalities have been utilized to navigate zero-contrast PCl. Real-time angiographic co-registration of IVUS images is very helpful in such interventions. This technique can be used without contrast injection and allows merging of the probe position on the guidewire with IVUS pictures, i.e., "dry" angiographic co-registration of IVUS. IVUS was also combined with near-infrared spec- 
troscopy (NIRS) to determine plaque composition during zero-contrast $\mathrm{PCl}$ of saphenous vein grafts, which proved to be useful in deciding whether to use a embolic protection device [20]. In several reports, optical coherence tomography (OCT) with angiographic co-registration was employed to guide the procedure [8, 21, 22]. Since the contrast medium could not be used for blood flushing during OCT, it was replaced by a mixture of saline and colloid or dextran-40 - these solutions provided sufficient image quality in OCT and had a good safety profile. After $\mathrm{PCl}$, repeated co-registered OCT determined the minimal stent area and guided post-dilation. These cases highlight the feasibility of radio-contrast free OCT as an imaging option for guiding zero-contrast $\mathrm{PCI}[8,21,22]$.

\section{Safety measures}

As in any other invasive coronary procedures, it is mandatory to monitor hemodynamics throughout the interventions. Even though zero-contrast $\mathrm{PCl}$ seems to be safe in experienced hands, it may, however, be associated with serious complications. Therefore, it is advisable to perform such procedures only for large and prognostically or clinically relevant coronary vessels. The risk of complications is probably higher when the target vessel diameter is small. In such circumstances, incorrect wiring of small side branches is very likely, and if unrecognized, may lead to perforation. Indeed, procedures without contrast may potentially miss coronary perforation; hence baseline and post- $\mathrm{PCl}$ echocardiography should be performed to check for pre-existing pericardial effusion and new or enlarging effusion after $\mathrm{PCl}$. Due to legal issues, it is sensible to document the final $\mathrm{PCI}$ result with a single injection of a small contrast volume. The amount of 2-3 $\mathrm{ml}$ of contrast should not pose a problem for renal function, but may reveal initial signs of complications, e.g., perforation and distal embolization - such an approach allows early bail-out management. Some other situations may require radio-contrast angiography during zero-contrast $\mathrm{PCl}$, i.e., persistent chest pain, new electrocardiographic changes, hemodynamic compromise, lack of improvement in physiological indices (i.e., FFR, (FR) and new or enlarging pericardial effusion [7]. Even in such conditions, contrast volume should be as low as possible, but it still must be sufficient to manage complications effectively. Therefore, before making the decision of performing zero-contrast $\mathrm{PCl}$, its potential benefit, anatomical conditions and patient's preferences must be considered.

\section{Adequate patient's hydration in zero-contrast interventions}

Although contrast volume reduction plays a critical role in preventing $\mathrm{CIN}$, adequate patient's hydration remains the cornerstone for this prevention. In the POSEIDON trial, left ventricular end-diastolic pressure
(LVEDP) guided fluid administration proved to be very effective and a safe way to prevent CIN in patients undergoing cardiac catheterization [23]. According to this study, each patient should receive a bolus of saline infusion at $3 \mathrm{ml} / \mathrm{kg}$ for $1 \mathrm{~h}$ before the procedure. In the setting of the catheterization laboratory, a pigtail catheter should be placed into the left ventricle for the baseline LVEDP measurement. Then, the fluid rate administration is adjusted in line with LVEDP, i.e.: $5 \mathrm{ml} / \mathrm{kg} / \mathrm{h}$ for LVEDP lower than $13 \mathrm{~mm} \mathrm{Hg}, 3 \mathrm{ml} / \mathrm{kg} / \mathrm{h}$ for LVEDP of 13-18 mm Hg, and $1.5 \mathrm{ml} / \mathrm{kg} / \mathrm{h}$ for LVEDP higher than $18 \mathrm{~mm} \mathrm{Hg}$. Such a fluid rate is started at the beginning of the procedure and continued during the procedure and for the next $4 \mathrm{~h}$. If the patient's body mass is higher than $100 \mathrm{~kg}$, the bolus and infusion rate should be limited to those calculated for subjects weighing $100 \mathrm{~kg}$. Such a management in the POSEIDON trial significantly reduced the incidence of CIN compared with the standard protocol (i.e., 6.7\% vs. 16.3\%) [23], and may constitute an excellent supplementary treatment in zero-contrast $\mathrm{PCl}$.

\section{Current clinical evidence}

Ultra-low contrast coronary angiography and zerocontrast $\mathrm{PCl}$ are new approaches in the prevention of $\mathrm{CIN}$; therefore, clinical data regarding their feasibility, efficacy and safety are limited. The first description of the method with presentation of 4 cases was published by Nayak et al. in 2010. PCls were performed ad hoc with no complications and none of the patients developed CIN [6]. However, the first substantial evidence of the feasibility and efficacy of zero-contrast $\mathrm{PCl}$ in the prevention of CIN was provided by Ali et al., who performed diagnostic angiography using ultra-low contrast volume followed by staged zero-contrast $\mathrm{PCl}$ in 31 patients with advanced CKD (mean eGFR: $16 \mathrm{ml} / \mathrm{min} / 1.73 \mathrm{~m}^{2}$ ) [7]. The median contrast volume during angiography was $13 \mathrm{ml}$ (median CV/eGFR ratio: 0.8) and a week later patients underwent $\mathrm{PCl}$ guided only by IVUS imaging and measurements of physiological indices (i.e., FFR and CFR). The median intervention time was $72 \mathrm{~min}$, median radiation dose was 1154 mGy, no procedural complications occurred, and no patient developed CIN. The same group published a series of 3 patients who underwent zero-contrast PCI facilitated with rotational atherectomy [9]. This report highlights the feasibility of performing rotational atherectomy as a part of zero-contrast $\mathrm{PCl}$ in selected patients with severe coronary calcifications.

There are several single case reports documenting PCI with no contrast for different anatomical settings, including complex left main stenosis, saphenous vein graft obstruction, multivessel disease and chronic total occlusion $[10,12,19,20,24]$. All of these cases were performed as staged procedures, none of these subjects developed $\mathrm{CIN}$ and no complications were reported. However, publication bias is likely since operators usually do not re- 
port failed or complicated cases. In several reports, OCT without contrast was used as a primary imaging modality; nevertheless, the majority of zero-contrast PCls were guided by IVUS. Due to its simplicity and wide availability, IVUS imaging appears to be the preferred method. Strong evidence that IVUS-guided $\mathrm{PCI}$ is safe and significantly reduces contrast dose was provided by the MOZART trial where 83 patients were randomly assigned to angiography-guided $\mathrm{PCI}$ or IVUS-guided PCI [25]. The IVUS approach was associated with a 3-fold reduction in contrast dose compared with the angiography arm (median volumes: $20.0 \mathrm{ml}$ vs. $64.5 \mathrm{ml}$, respectively) and it was not related to any specific complications. However, the study was not powered to detect differences in hard endpoints, and most of the subjects presented relatively good renal function; hence, no clinical benefit (including reduction of CIN) was observed [24].

Recently, one prospective study and another retrospective analysis showed that ultra-low contrast techniques guided by only angiography (without IVUS and OCT) is feasible and can safely be used in patients with CKD $[26,27]$. These single arm studies showed that contrast dose might be reduced to a median volume of 13 to $15.2 \mathrm{ml}$ for diagnostic angiography and 13 to $17.1 \mathrm{ml}$ for $\mathrm{PCl}$, which was associated with a low rate of CIN [26, 27]. Although this approach is cheaper compared with IVUS- or OCT-guided procedures, there are some concerns about its long-term outcomes, i.e., whether ultra-low contrast PCI without intravascular imaging ensures optimal results. Therefore, studies with long-term follow-up are needed to check clinical outcomes of this approach.

It should also be established whether staged procedures, after minimal contrast diagnostic angiography, present any benefit over ad hoc $\mathrm{PCl}$. Deferral of the intervention facilitates procedural planning and enables us to discuss all treatment options with patients as well as obtain their informed consent [7]. In a study by Ali et al., staged $\mathrm{PCl}$ was performed no earlier than 7 days after angiography, i.e., when the contrast agent is expected to be completely excreted by the kidneys; the authors argue that their excellent results with no CIN incidents prove the appropriateness of such an approach [7, 28]. Deferred $\mathrm{PCl}$ permits optimal patient hydration, which is a critical element in CIN prevention, including zero-contrast interventions $[1,23]$. However, in our recent study, 13 patients with acute coronary syndrome underwent zero-contrast $\mathrm{PCl}$ and 4 of them suffering from non-STsegment elevation myocardial infarction were treated with ad hoc procedures with very good clinical outcomes [29]. Thus, regardless of the mode of intervention (ad hoc or staged), subjects with severe renal impairment seem to benefit from zero-contrast $\mathrm{PCl}$. Yet, due to the need of adequate hydration, a staged approach should rather be standard in most cases except those with acute coro- nary syndromes where the clinical context must be taken into account when making a decision about the time of intervention.

A new concept for the use of the zero-contrast approach is the protection of residual renal function in hemodialysis patients undergoing coronary interventions $[12,29]$. It has been demonstrated that the residual renal function is a prognostic and independent factor of quality of life, morbidity and survival in dialysis patients, and therefore every protective measure to preserve this function is worth considering [30]. In our recent study, 4 dialysis subjects were treated with zero-contrast $\mathrm{PCI}$ and 3 of them preserved their remaining renal function [29]. However, despite this promising observation, the concept should be addressed in appropriate prospective studies before it is recommended for wide clinical practice.

\section{Conclusions}

Contrast-induced nephropathy is a common complication after diagnostic and therapeutic coronary procedures and is independently associated with adverse outcomes. Although many therapies have been tested to avoid CIN, the only potent preventative strategy involves aggressive fluid administration and reduction of contrast volume. Approaches aiming to minimize contrast dose have become new and effective weapons in the battle with CIN. However, despite the first promising results, they still require further development and confirmation of their effectiveness as well as safety in large clinical studies. Advances in imaging techniques will certainly facilitate such interventions and should cause a growth of interest in these approaches. However, to adopt contrast limiting techniques, invasive cardiologists must change their routines in daily practice. First, during both angiography and $\mathrm{PCl}$, they ought to seek anatomical landmarks (particularly calcifications) to navigate their interventions without contrast usage. Second, small injections with small syringes as well as evacuation of unused contrast from catheters must be standard. Finally, wider use of intravascular imaging techniques (especially IVUS) should decrease contrast volume and improve $\mathrm{PCl}$ results. This article summarizes the principles of these approaches and should help in spreading them among the interventional community.

\section{Conflict of interest}

The authors declare no conflict of interest.

\section{References}

1. Azzalini L, Spagnoli V, Ly HQ. Contrast-Induced nephropathy: from pathophysiology to preventive strategies. Can J Cardiol 2015; 32: 247-55.

2. Mehran R, Aymong ED, Nikolsky E, et al. A simple risk score for prediction of contrast-induced nephropathy after percutaneous 
coronary intervention: development and initial validation. J Am Coll Cardiol 2004; 44: 1393-9.

3. Tsai TT, Patel UD, Chang TI, et al. Contemporary incidence, predictors, and outcomes of acute kidney injury in patients undergoing percutaneous coronary interventions: insights from the NCDR Cath-PCI registry. JACC Cardiovasc Interv 2014; 7: 1-9.

4. Katzberg RW, Barrett BJ. Risk of iodinated contrast material-induced nephropathy with intravenous administration. Radiology 2007; 243: 622-8.

5. Owen RJ, Hiremath S, Myers A, et al. Canadian Association of Radiologists consensus guidelines for the prevention of contrast-induced nephropathy: update 2012. Can Assoc Radiol J 2014; 65: 96-105.

6. Nayak KR, Mehta HS, Price MJ, et al. A novel technique for ultra-low contrast administration during angiography or intervention. Catheter Cardiovasc Interv 2010; 75: 1076-83.

7. Ali ZA, Karimi Galougahi K, Nazif T, et al. Imaging- and physiology-guided percutaneous coronary intervention without contrast administration in advanced renal failure: a feasibility, safety, and outcome study. Eur Heart J 2016; 37: 3090-5.

8. Karimi Galougahi K, Zalewski A, Leon MB, et al. Optical coherence tomography-guided percutaneous coronary intervention in pre-terminal chronic kidney disease with no radio-contrast administration. Eur Heart J 2016; 37: 1059.

9. Karimi Galougahi K, Mintz GS, Karmpaliotis D, Ali ZA. Zero-contrast percutaneous coronary intervention on calcified lesions facilitated by rotational atherectomy. Catheter Cardiovasc Interv 2017; 90: E85-9.

10. Sacha J, Feussette P. How should I treat unprotected LM stenosis in a patient with ACS who develops a severe contrast-induced nephropathy after coronary angiography? https://www. pcronline.com/Cases-resources-images/Cases/EIJ-HSIT/Treatment-strategy-for-left-main-stenosis-in-patient-with-CIN?auth=true\#comments_area (Published, 06 December 2017).

11. Sacha J. Marking wire technique for zero-contrast percutaneous coronary interventions. Adv Interv Cardiol 2018; 14: 204-5.

12. Sacha J, Feussette P, Gierlotka M. Full triple-vessel coronary revascularization with ultra-low contrast administration as a strategy for preserving residual renal function in patient with acute coronary syndrome. Kardiol Inwazyjna 2018; 13: 33-40.

13. Lai J, Akindavyi G, Fu Q, et al. Research progress on the relationship between coronary artery calcification and chronic renal failure. Chin Med J (Engl) 2018; 131: 608-14.

14. Kim JK, Kim NH, Shin IS, et al. Alteration of ventricular repolarization by intracoronary infusion of normal saline in patients with variant angina. Korean Circ J 2009; 39: 223-7.

15. Jo SH, Youn TJ, Koo BK, et al. Renal toxicity evaluation and comparison between visipaque (iodixanol) and hexabrix (ioxaglate) in patients with renal insufficiency undergoing coronary angiography: The RECOVER study: a randomized controlled trial. J Am Coll Cardiol 2006; 48: 924-30.

16. McCullough PA, Bertrand ME, Brinker JA, Stacul F. A meta-analysis of the renal safety of isosmolar iodixanol compared with low-osmolar contrast media. J Am Coll Cardiol 2006; 48: 692-9.

17. Sherwin PF, Cambron R, Johnson JA, Pierro JA. Contrast doseto-creatinine clearance ratio as a potential indicator of risk for radiocontrast-induced nephropathy: correlation of $\mathrm{D} / \mathrm{CrCL}$ with area under the contrast concentration-time curve using iodixanol. Invest Radiol 2005; 40: 598-603.

18. Kane GC, Doyle BJ, Lerman A, et al. Ultra-low contrast volumes reduce rates of contrast-induced nephropathy in patients with chronic kidney disease undergoing coronary angiography. J Am Coll Cardiol 2008; 51: 89-90.

19. Sacha J, Feusette P. Zero-contrast percutaneous coronary intervention of saphenous vein graft in a patient with chronic renal failure. Adv Interv Cardiol 2018; 14: 309-311.

20. Parviz Y, Fall K, Stone GW, et al. Imaging and physiology to guide venous graft Interventions without contrast administration in advanced renal failure. J Invasive Cardiol 2017; 29: E163-5.

21. Azzalini L, Mitomo S, Hachinohe D, et al. Zero-contrast percutaneous coronary intervention guided by dextran-based optical coherence tomography. Can J Cardiol 2018; 34: 342.e1-3.

22. Azzalini L, Hachinohe D, Regazzoli D, Colombo A. Ultra-low contrast percutaneous coronary intervention in patients with severe chronic kidney disease. Eurointervention 2018; 14: 896-7.

23. Brar SS, Aharonian V, Mansukhani P, et al. Haemodynamic-guided fluid administration for the prevention of contrast-induced acute kidney injury: the POSEIDON randomised controlled trial. Lancet 2014; 383: 1814-23.

24. Hatem R, Finn MT, Riley RF, et al. Zero contrast retrograde chronic total occlusions percutaneous coronary intervention: a case series. Eur Heart J Case Rep 2018; 2: 1-5.

25. Mariani J Jr, Guedes C, Soares P, et al. Intravascular ultrasound guidance to minimize the use of iodine contrast in percutaneous coronary intervention: the MOZART (Minimizing cOntrast utiliZation With IVUS Guidance in coRonary angioplasTy) randomized controlled trial. JACC Cardiovasc Interv 2014; 7: 1287-93.

26. Rozenbaum Z, Benchetrit S, Rozenbaum E, et al. Ultra-low contrast volume for patients with advanced chronic kidney disease undergoing coronary procedures. Nephron 2018; 138: 296-302.

27. Kelly SC, Li S, Stys TP, et al. Reduction in contrast nephropathy from coronary angiography and percutaneous coronary intervention with ultra-low contrast delivery using an automated contrast injector system. J Invasive Cardiol 2016; 28: 446-50.

28. Lorusso V, Taroni P, Alvino S, Spinazzi A. Pharmacokinetics and safety of iomeprol in healthy volunteers and in patients with renal impairment or end-stage renal disease requiring hemodialysis. Invest Radiol 2001;36:309-16 20.

29. Sacha J, Gierlotka M, Lipski P, et al. Zero-contrast percutaneous coronary interventions to preserve kidney function in patients with severe renal impairment and hemodialysis subjects. Adv Interv Cardiol 2019; 15: 137-42.

30. van der Wal WM, Noordzij M, Dekker FW, et al. Full loss of residual renal function causes higher mortality in dialysis patients; findings from a marginal structural model. Nephrol Dial Transplant 2011; 26: 2978-83. 